\title{
Effet des extraits aqueux de Calotropis procera sur les principaux ravageurs du chou en culture au Sénégal
}

\author{
Saliou NGOM*, Toffène DIOME, Bocar DIOP et Mbacké SEMBENE \\ Equipe de Génétique et de Gestion des Populations, Département de Biologie Animale, \\ Université Cheikh Anta Diop, Dakar, Sénégal. \\ *Auteur correspondant ; E-mail : salioungom5@gmail.com; Tel : +221775972636
}

\section{RESUME}

Au Sénégal, le chou est l'un des légumes les plus cultivés et consommés, de par son cycle relativement court (60-90 jours après repiquage) et sa possibilité d'être cultivé toute l'année. Cependant il est attaqué par plusieurs ravageurs tels que Plutella xylostella (L.) (Lepidoptera, Plutellidae), Hellula undalis (F.) (Lepidoptera, Pyralidae) et Spodoptera littoralis (B.) (Lepidoptera, Noctuidae). L'objectif de cette étude était de contribuer à la lutte contre ces ravageurs du chou en culture dans la zone des Niayes en utilisant les extraits aqueux à base de feuilles de Calotropis procera (Apocynaceae). L'étude a été menée sur 28 parcelles élémentaires dans la zone des Niayes (durant la période du 3 janvier au 22 mars 2019). Les formulations d'extrait de feuilles de C. procera utilisant comme solvant l'eau de robinet et l'eau de puits (ER et EP), appliquées à des temps différents le matin (ERM, EPM) et le soir (ERS, EPS) ont montré leurs efficacités sur les larves de P. xylostella et H. undalis. Par contre, les extraits ne sont pas efficaces sur $S$. littoralis $(\mathrm{P}$-value= 0,672). En plus de leurs effets biocides, les extraits à base de feuilles de $C$. procera semblent accélérer la croissance des plants de chou. La nature du solvant et la période du traitement n'ont pas montré une différence significative.

(C) 2020 International Formulae Group. All rights reserved.

Mots clés : Biocide, Brassicassée, Niayes, lutte biologique

\section{Aqueous extracts effect of Calotropis procera on the principal cabbage pests in culture in Senegal}

\begin{abstract}
In Senegal, the cabbage is one of the most cultivated and consumed vegetables, from its relatively short cycle (60-90 days after road repair) and the possibility of being cultivated all the year. However it is attacked by several pests such as Plutella xylostella (L.) (Lepidoptera, Plutellidae), Hellula undalis (F.) (Lepidoptera, Pyralidae) and Spodoptera littoralis (B.) (Lepidoptera, Noctuidae). The objective of this study was to contribute on protect against cabbage pests in culture in the zone of Niayes by using the aqueous extracts containing sheets of Calotropis procera (Apocynaceae). The study was undertaken on 28 elementary pieces to in the zone of Niayes (during the period of January 3 at March 22, 2019). The formulations of extract using as solvent the water of tap and the water of well (ER and EP), applied to different times morning (ERM, EPM) and evening (ERS, EPS) showed their effectiveness on larvae of $P$. xylostella and $H$. undalis. On the other part $S$. littoralis was resistant to treatment $(\mathrm{P}=0,672)$ confirms it. In addition to their biocides effects, the extracts containing sheets of $C$ procera seem accelerated the cabbage patches growth. The nature of solvent and the treatment period did not show a significant difference.

(C) 2020 International Formulae Group. All rights reserved.
\end{abstract}

Keywords: Biocide, Brassicasseae, Niayes, biological control. 


\section{INTRODUCTION}

Les cultures maraîchères occupent une place importante pour l'alimentation humaine et contribuent significativement aux revenus des familles en Afrique de l'Ouest, mais leur production est confrontée à une pression des bio agresseurs qui limite leur productivité (Yarou et al., 2017). La culture des Brassicacées est l'une des productions agricoles les plus importantes au monde (Arvanitakis, 2013). D'après les données de la FAO (FAOSTAT, 2013), 37 millions d'hectares ont été cultivés en 2011 avec une production annuelle globale de 152 millions de tonnes uniquement pour le chou, le chou-fleur et le colza. Au Sénégal, le chou fait partie des légumes les plus cultivés et consommés, de par son cycle relativement court (60-90 jours après repiquage) et la possibilité d'être cultivé toute l'année (Labou, 2016). Il est le second pays producteur de chou en Afrique de l'ouest, après le Niger (Labou, 2016). Cependant, sa production est sérieusement affectée par un nombre important de ravageurs dont les principaux sont: la teigne du chou, Plutella xylostella (Lepidoptera, Plutellidae), Hellula undalis (Lepidoptera, Pyralidae) et Spodoptera litoralis (Lepidoptera, Noctuidae) (Mondedji et al., 2014). Au Sénégal, la teigne du chou a une incidence largement supérieure à celle de l'espèce $H$. undalis car elle est présente partout où pousse des plants de chou (Labou, 2016). La présence des chenilles et chrysalides de la teigne sur les plantes de chou se matérialise par la perforation des feuilles. Selon Diomaha et Ghogomu (2016), le pourcentage de surface foliaire total endommagée par $P$. xylostella peut varier de 0 à 50\%. Pour limiter les dégâts des ravageurs et améliorer la production en Afrique, les maraîchers utilisent principalement des produits de synthèse (Goalbaye et al., 2016). Bien que les programmes « champs écoles » initiés aient permis de réduire leur usage, les problèmes liés à leur utilisation demeurent toujours. Ainsi les pesticides de synthèse sont généralement néfastes aux auxiliaires (prédateurs et parasitoïdes) des ravageurs (Silva-Torres et al.,
2010) et à la santé humaine. La résistance aussi des bioagresseurs est l'une des principales limites de ces derniers. Celle aux pyréthrinoïdes, organochlorés, organophosphorés et carbamates a été notifiée par exemple sur P. xylostella (Gnago et al., 2011; Guèye et al., 2011; Arvanitakis, 2013; Mawussi et al., 2014). En plus de cette résistance, des résidus de substances actives sont détectés dans divers produits maraîchers, parfois à des quantités dépassant les limites maximales de résidus (LMR) fixées par le Codex Alimentarius ou l'Union Européenne (UE) (Son et al., 2017; Yarou et al., 2017). De l'endosulfan et du diméthoate ont été détectés dans les eaux de puits en zone agricole au Sénégal (Yarou et al., 2017). La pollution environnementale, quant à elle, résulte aussi bien des effets de surdosage que de la mauvaise gestion des emballages (Goalbaye et al., 2016). Pour remédier aux problèmes résultants de l'utilisation des pesticides de synthèse (Boisclair et Estevez, 2006), les plantes pesticides se présentent comme une alternative prometteuse dans le contexte de l'Afrique de l'Ouest. L'azadirachtine a montré une grande efficacité en terme de production de choux commercialisables pour lutter contre les ravageurs (Daly et al., 2000). L'extrait brut des feuilles de Calotropis procera a montré une forte toxicité à l'égard de Caryedon serratus (Thiaw, 2008). C'est dans ce contexte que ce travail a été initié pour une lutte efficace et respectueuse de l'environnement contre les ravageurs du chou par usage de biopesticides. L'utilisation des plantes pesticides se révèle être une pratique ancestrale en Afrique. En effet, de nombreuses plantes sont connues et utilisées pour leurs activités biocides (toxique, répulsive, anti-appétant) vis-à-vis d'une large gamme de bioagresseurs. Elles peuvent être utilisées sous forme d'extraits de plantes en protection foliaire (Yarou et al., 2017). Le but de notre étude était de tester l'effet biocide des extraits aqueux de $C$. procera sur les principaux ravageurs du chou dans la zone des Niayes. 


\section{MATERIEL ET METHODES}

\section{Présentation de la zone d'étude}

Située le long du littoral nord du Sénégal, la zone des Niayes est une bande de terre de $180 \mathrm{~km}$ de long sur une vingtaine de kilomètres de large allant de Dakar à St-Louis avec une largeur variant de 5 à $30 \mathrm{~km}$ ( Fall et al., 2001; Touré et Seck, 2005; Ngom et al., 2012). Le climat dans les Niayes est de type tropical sub canarien avec une humidité relativement élevée. Une faune et une flore très variées. L'analyse pétrographique montre que les Niayes sont constituées de limon fin et grossier, de sable fin et grossier et d'argile (Mbengue, 2011). Dans cette zone, l'eau disponible et accessible provient de la nappe souterraine et les eaux de surface localisées au niveau d'un certain nombre de lacs.

\section{Extraction de la substance biocide}

Les organes végétaux utilisés sont des feuilles de $C$. procera récoltées avant le lever le du soleil. Les feuilles étaient fraîches à température ambiante. Après pesage, elles sont broyées par un mixeur, mises en macération dans un seau pendant 24 heures à une concentration de $400 \mathrm{~g}$ par litre. Les solvants utilisés pour l'extraction de la substance biocide sont l'eau de robinet (ER) et l'eau de puits (EP). Le mélange est filtré à l'aide d'un tamis à mailles fines $(0,01 \mathrm{~mm} \times 0,01 \mathrm{~mm})$. Le produit fini était conservé à température ambiante.

\section{Dispositif expérimental}

La mise en place de la culture de chou variété de saison sèche de type «Calif $\mathrm{F} 1$ »a été faite en collaboration avec les maraîchers. Une parcelle de $28 \mathrm{~m}^{2}$ divisée en parcelles élémentaires distantes de $70 \mathrm{~cm}$ entre les blocs a été utilisée. Chacune des parcelles élémentaires $(1 \mathrm{~m} \times 1 \mathrm{~m})$ portait 2 rangées de 3 pieds de chou espacés de $40 \mathrm{~cm}$ sur les lignes et $35 \mathrm{~cm}$ entre les lignes. Quatre types de traitement ont été appliqués (traitement le matin avec un solvant eau de puits et eau de robinet; un traitement le soir avec un solvant eau de puits et eau de robinet) et un témoin.
Chaque traitement correspond à une parcelle élémentaire. Les parcelles élémentaires étaient disposées dans cinq blocs, donc cinq répétitions chacune.

\section{Techniques culturales Semis et pépinière}

Les activités avaient commencé le jeudi 03 janvier 2019 avec le semis du chou. Des alvéoles ont été utilisés comme support et du terreau pour la fertilisation. Durant les trois semaines correspondant à la durée de la pépinière, un diagnostic s'effectuait deux jours par semaine pour observer s'il y'avait apparition de maladie ou de grains non germés.

\section{Repiquage au champ}

Le champ a été aménagé le mercredi 23 janvier 2019 puis le lendemain, toute la surface a été arrosée, soit 3 arrosoirs de $10 \mathrm{~L}$ par parcelle élémentaire. Après quelques minutes d'attente pour que le sol s'humidifie, les écarts entre les pieds de chou étaient mesurés en prenant des écartements de $40 \mathrm{~cm}$ soit 6 pieds par parcelle élémentaire. Le repiquage des pieds de chou a été effectué le même jour. Ainsi, $10 \mathrm{~L}$ d'eau sont encore appliqués dans chaque parcelle élémentaire.

\section{Traitement phytosanitaire}

Les traitements phytosanitaires ont été réalisés avec des extraits aqueux de feuilles fraîches de $C$. procera à une concentration de $400 \mathrm{~g} / \mathrm{l}$ pour qu'il n'y ait de variation que sur la période et la nature du solvant, avec un mélange eau de puits- $C$. procera (EPC) et un mélange eau de robinet- $C$. procera (ERC). Une fois au champ, les extraits aqueux étaient appliqués au niveau des plants à l'aide d'un pulvérisateur de 201 . Cette application avait commencé trois jours après repiquage. Le traitement s'effectuait tous les 15 jours par pulvérisation jusqu'au 15ème jour avant la récolte et des relevés sur le nombre s'effectuaient deux jours avant et après chaque traitement afin de suivre l'évolution des larves. Dans les blocs, chaque parcelle élémentaire 
correspondait à un traitement excepté le témoin.

\section{Paramètres étudiés}

Des relevés sur le nombre s'effectuaient deux jours avant et après chaque traitement afin de suivre l'évolution des larves

Les paramètres de la diversité écologique suivants ont été déterminés :

\section{Richesse en espèces des parcelles}

Effectif par espèce $=\mathbf{n i}$;

Fréquence d'occurrence $=\mathbf{S e} / \mathbf{S t}$ avec $\mathrm{Se}$ (sortie présentant l'espèce) et $\mathrm{St}$ (sortie total)

Incidence $=\mathbf{P i} / \mathbf{P t}$ avec $\mathrm{Pi}$ (nombre de pieds infestés par une espèce donnée) et $\mathrm{Pt}$ (nombre total des pieds repiqués).

\section{Abondance relative}

Elle est définie comme étant le rapport entre l'effectif de l'espèce i par exemple (ni) et l'effectif total des individus des différentes espèces du peuplement $(\mathbf{N})$ :

\section{$\boldsymbol{P i}=\boldsymbol{n i} / \mathbf{N}$}

L'étude de la diversité du peuplement a été réalisée à l'aide de nombreux indices :

\section{Indice de diversité de Shannon $H^{\prime} \alpha=-\Sigma P i \log 2 P i$}

$P i$ représente l'abondance relative.

L'indice $\boldsymbol{H}^{\prime} \boldsymbol{\alpha}$ permet de comparer la diversité de différentes communautés. L'indice de Shannon est issu de la théorie de l'information et sert au calcul de la diversité des signaux véhiculés par un canal. Il est appliqué avec succès à l'évaluation de la diversité dans les communautés. Les espèces y prennent la valeur des signaux.

\section{Indice de Simpson : IS $=\mathbf{1} / \mathbf{\Sigma} P \boldsymbol{i}^{2}$}

Il peut être interprété comme la probabilité que deux individus tirés au hasard soient d'espèces différentes. Il est compris dans l'intervalle [0,1[. Sa valeur diminue avec la régularité de la distribution. La valeur 1 est atteinte pour un nombre infini d'espèces, de probabilités nulles.

\section{Analyses des données}

Le tableur Microsoft Excel 2007 a été utilisé pour classer les données obtenues au terrain et son tableau de dynamique croisée pour générer les histogrammes. L'analyse des résultats a été faite avec le logiciel $\mathrm{R}$ version (3.5.3). L'effet du traitement sur les larves des espèces étudiées et sur le poids de la récolte a été déterminé avec le test de Kruskal wallis. Il s'achève avec le test de comparaison deux à deux de Wilcoxon vu que nos données ne suivaient pas une loi normale. Dans tous les cas, les tests seront considérés comme significatifs quand la probabilité de la p-value est inférieure au seuil fixé à 5\% $(0,05)$.

\section{RESULTATS}

Inventaire des espèces rencontrées dans le milieu

\section{Abondance}

Les ravageurs identifiés lors de nos échantillonnages appartiennent à l'ordre des lépidoptères, hémiptères, orthoptère et des diptères. Durant toute la durée de l'essai, 175 individus répartis en 5 espèces ont été recensés. L'espèce majoritaire était $S$. litoralis, 78 individus avec une abondance de $45 \%$ suivis respectivement de $P$. xylostella, 68 individus (39\%), de l'arpenteuse verte, $C$. chalcites avec 13 individus (7\%), la noctuelle de la tomate, $H$. armigera avec 8 individus (5\%) et le borer du chou, H. undalis avec 8 individus (5\%) (Tableau 1). Les pucerons aptères et ailés qui, classés en intervalle par défaut de comptage précis, étaient très abondants dans le milieu tout comme leurs prédateurs.

\section{Fréquence d'occurrence et l'incidence des espèces étudiées}

Les espèces $S$. litoralis, $P$. xylostella et C. chalcites présentent à cette période une fréquence d'occurrence de $80 \%$ (Tableau 2) ce qui signifie qu'ils étaient constants dans le milieu. $P$. xylostella et $C$. chalcites ont été répertoriés dès le $3^{\text {ième }}$ jour de repiquage et présentaient respectivement une incidence de $35,71 \%$ et $14,29 \%$, tandis que $S$. litoralis n'a été observée qu'au $12^{\text {ième }}$ jour après repiquage avec une incidence de $46,43 \%$. Le Borer du chou (H. undalis) et la noctuelle $H$. armigera quant à eux étaient détectés dans $40 \%$ des parcelles élémentaires avec une incidence de 
$10,71 \%$ chacune. Malgré la faible incidence du borer, des dégâts au niveau de deux bourgeons terminaux ont été notés causant la formation de pommes supplémentaires. Les dégâts agronomiques sont plus importants pour Spodoptera que les autres ravageurs.

\section{Diversité des espèces}

L'analyse du Tableau 3 des indices montre une très grande diversité dans les différentes parcelles élémentaires (PE) avec une valeur de l'indice de Shannon ( $\left.H^{\prime} \alpha=1,74\right)$. Plutella xylostella et $S$. littoralis étaient plus représentés dans les différentes $\mathrm{PE}$ avec les valeurs de $H^{\prime} \alpha 0,53$ et 0,52 respectivement. Crysodeixis chalcites quand à lui a une valeur H'a: 0,28. Le borer Hellula undalis et la noctuelle $H$. armigera étaient faiblement représentés avec une valeur de $\mathrm{H}^{\prime} \alpha(0,20)$ chacun. La valeur de l'indice de Simpson $(2,78)$ vient confirmer qu'on a un bon degré de biodiversité des ravageurs.

\section{Effet du traitement sur les larves des espèces étudiées}

L'analyse de la Figure 1 représentant le nombre de larves des espèces selon les traitements révèle que pour $P$. xylostella $(\mathrm{Px})$ et H. undalis $(\mathrm{Hu})$, il était nul après chaque traitement (Post Traitement) correspondant au $5^{\text {ième }}$ jour de la semaine. Cependant leurs présences étaient toujours notées avant le traitement (AT) durant les trois premières semaines mais à la $4{ }^{\text {ième }}$ semaine, leurs nombres étaient pratiquement tous nul. L'effet du traitement est significatif sur P. xylostella et $H$. undalis avec des P-value de 0,004 et 0,024 respectivement en les diminuant.

Le nombre de larves de S. littoralis ( $\mathrm{Sl}$ ) augmente depuis son apparition correspondant à la $2^{\text {ième }}$ semaine du traitement. Cependant, l'effet du traitement n'est pas significatif (Pvalue $=0,672$ ). Une forte augmentation $d u$ nombre de larve de $P$. xylostella à partir de la $5^{\text {ième }}$ semaine correspondant à l'arrêt du traitement (ArT) a été notée. De même que $H$. undalis au $5^{\text {ième }}$ jour (ArT) bien qu'elle était l'espèce la moins représentée.

\section{Effet du solvant et de la période de traitement sur les larves}

Le test de comparaison deux à deux n'a montré aucune significativité avec $P$. xylostella entre les formulations Eau de Puits Matin (EPM) et Eau de Robinet Matin (ERM) (Pvalue $=0,827$ ) d'une part et entre Eau de Puits Soir-Eau de Robinet Soir (EPS-ERS) (Pvalue $=0,42$ ) d'autre part. Par contre, une différence significative est notée entre les autres formulations Eau de Puits MatinTémoin $($ EPM-T $)(\mathrm{P}$-value $=0,031)$, EPS-T $(\mathrm{P}-$ value $=0,004)$ et ERS-T $(P-$ value $=0,037)$. Pour l'espèce $H$. undalis, on note une différence significative entre toutes les formulations (ERM-T $:$ P-value $=0,04$ et EPM-T $: \mathrm{P}$-value= 0,04) sauf EPS-T (P-value=0,41). Avec l'espèce $S$. littoralis aucune différence significative n'est notée entre les différentes formulations EPM-ERM (P-value $=0,592)$, EPM-EPS $(\mathrm{P}$-value $=0,235)$ et ERM ERS $(\mathrm{P}$ value $=0,672)$ mais elle est notée entre ERM$\mathrm{T}(\mathrm{P}$-value $=0,001)$.

\section{Effet du traitement sur la qualité et la maturité des pommes}

Sur l'ensemble des plants repiqués, 2 plants étaient non commerçables à cause de leurs forte pommaille qui finissent par exploser. Ces pommes sont récoltées dans la $20^{\text {ième }}$ parcelle ayant reçu le traitement Puits Soir (PS) et dans la 14 ${ }^{\text {ième }}$ parcelle traitée par Puits Matin (PM). Après attaque précoce par $H$. undalis dans la $21^{\text {ième }}$ Parcelle Élémentaire (PE) traitée par PM, le plant a formé 3 pommes commerçables. De même que dans la $16^{\text {ième }} \mathrm{PE}$ correspondant au témoin avec la formation de 2 pommes commerçables. Nous avions donc à la fin 169 pommes commerçables. Toutes les parcelles ayant reçu de traitement ont été récoltées au 75ième jour mais le témoin est récolté tardivement, 15 jours après, correspondant au $90^{\text {ième }}$ jour. 
Evolution des larves des différents ravageurs en fonction du traitement

L'analyse de la Figure 2 montre l'évolution du nombre de larve en fonction du traitement. A la première semaine $(\mathrm{S} 1)$ de traitement, seules les larves de $P$. xylostella colonisaient la parcelle. De la $2^{\text {ième }}$ à la $3^{\text {ième }}$ semaine (S2, S3), sa population diminuait jusqu'à s'annuler après chaque traitement avec Post Traitement. Nous avions noté qu'à la $4^{\text {ième }}$ semaine (S4), sa population était nulle du $1^{\text {ier }}$ jour de traitement (J1) jusqu'au début de la $5^{\text {ième }}$ semaine (5) correspondant à l'arrêt du traitement (ArT). Par contre, les larves de $S$. littoralis n'étaient présentes qu'au $1^{\text {ier }}$ jour (J1) de la $\mathrm{S} 1$ et depuis lors sa population ne faisait qu'augmenter malgré les traitements appliqués. Les larves de $H$. undalis n'étaient présentes qu'à la $3^{\text {ième }}$ semaine $(\mathrm{S} 3)$ mais sa population était nulle au moment du traitement jusqu'au $3^{\text {ième }}$ jour (J3) de la S5 correspondant à l'arrêt du traitement (ArT). A partir de cette semaine, sa population commence à s'accroître.

Tableau 1 : Abondance des espèces étudiées.

\begin{tabular}{|c|c|c|c|c|c|c|}
\hline Espèces & $\begin{array}{l}P . \\
\text { xylostella }\end{array}$ & $\begin{array}{l}\text { H. } \\
\text { undalis }\end{array}$ & $\begin{array}{l}. \\
\text { littoralis }\end{array}$ & $\begin{array}{l}\text { H. } \\
\text { armigera }\end{array}$ & $\begin{array}{l}\text { C. } \\
\text { chalcites }\end{array}$ & Total \\
\hline Effectif (ni) & 68 & 8 & 78 & 8 & 13 & 175 \\
\hline Abondance relative $(\mathrm{Pi})$ & $39 \%$ & $5 \%$ & $45 \%$ & $5 \%$ & $7 \%$ & $100 \%$ \\
\hline
\end{tabular}

Tableau 2: Fréquences d'occurrence et l'incidence des différentes espèces étudiées.

\begin{tabular}{lllll}
\hline ORDRES & FAMILLES & $\begin{array}{l}\text { ESPECES/Noms } \\
\text { scientifiques }\end{array}$ & VARIABLES & MOYENNES (\%) \\
\hline & Plutellidae & P. xylostella & occurrence & $80,00 \%$ \\
& & incidence & $35,71 \%$ \\
Lepidoptera & Pyralidae & H. undalis & occurrence & $40,00 \%$ \\
& & & incidence & $10,71 \%$ \\
& & & occurrence & $80,00 \%$ \\
& S. littoralis & incidence & $46,43 \%$ \\
& & & occurrence & $40,00 \%$ \\
& & & incidence & $10,71 \%$ \\
& & & occurrence & $80,00 \%$ \\
& & & incidence & $14,29 \%$ \\
\hline
\end{tabular}


Tableau 3 : Indices de diversité des ravageurs.

\begin{tabular}{lll}
\hline Espèces & $(\mathbf{P i})^{\mathbf{2}}$ & $\mathbf{P i} \mathbf{L o g}_{2} \mathbf{P i}$ \\
\hline Espèces & 0,15 & $-0,53$ \\
P. xylostella & 0,00 & $-0,20$ \\
H. undalis & 0,20 & $-0,52$ \\
S. littoralis & 0,00 & $-0,20$ \\
H. armigera & 0,01 & $-0,28$ \\
C. Chalcites & 0,36 & $-1,74$ \\
Total $\left(\Sigma \mathrm{pi}^{2} ; \Sigma-\mathrm{H}^{\prime} \alpha\right)$ & 2,78 & \\
Is $=1 / \Sigma_{\mathrm{P}_{\mathrm{i}}}{ }^{2}($ Simpson) & & 1,74 \\
\hline
\end{tabular}
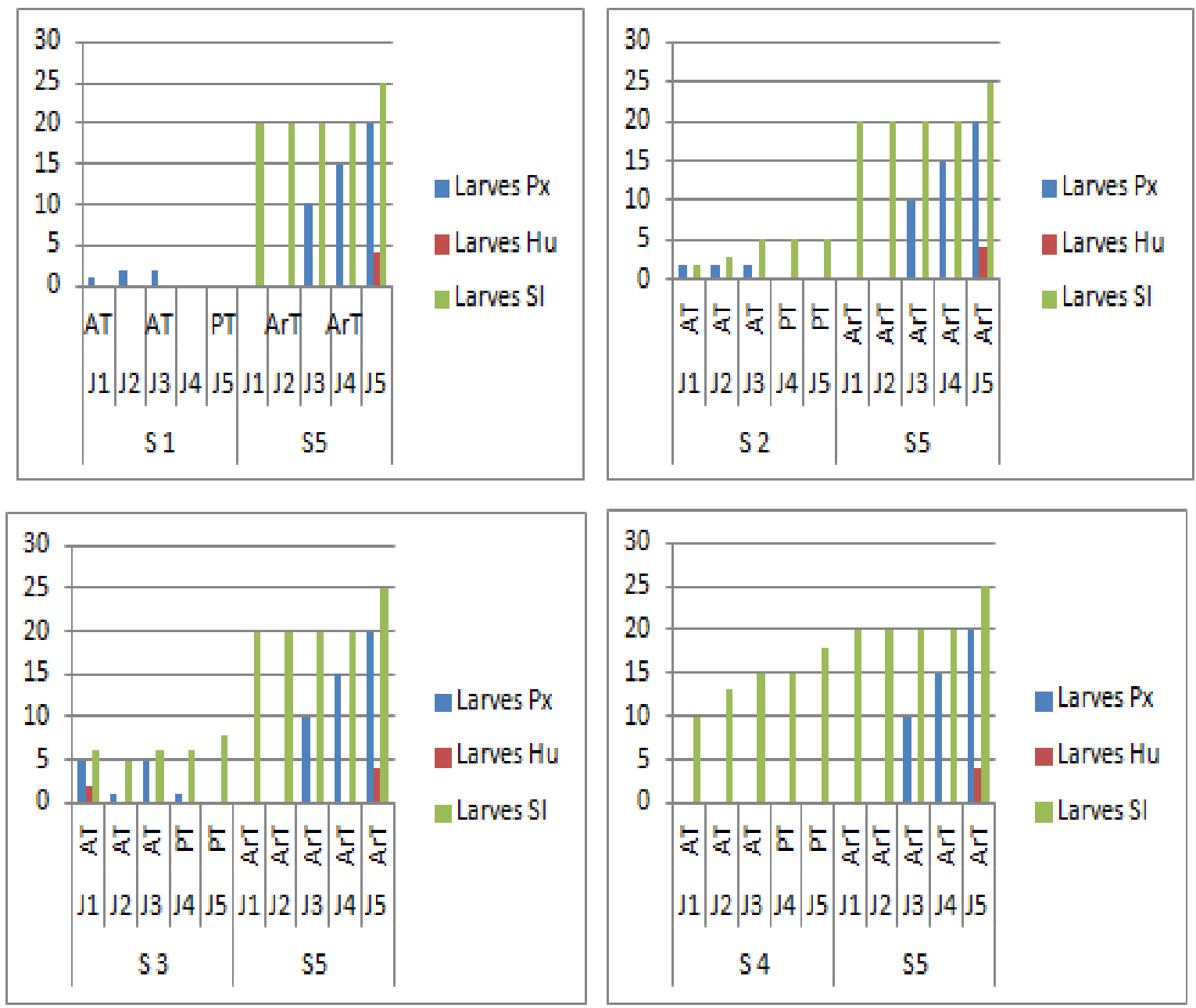

Figure 1: Comparaison du nombre de larves des espèces avant et après traitement. 


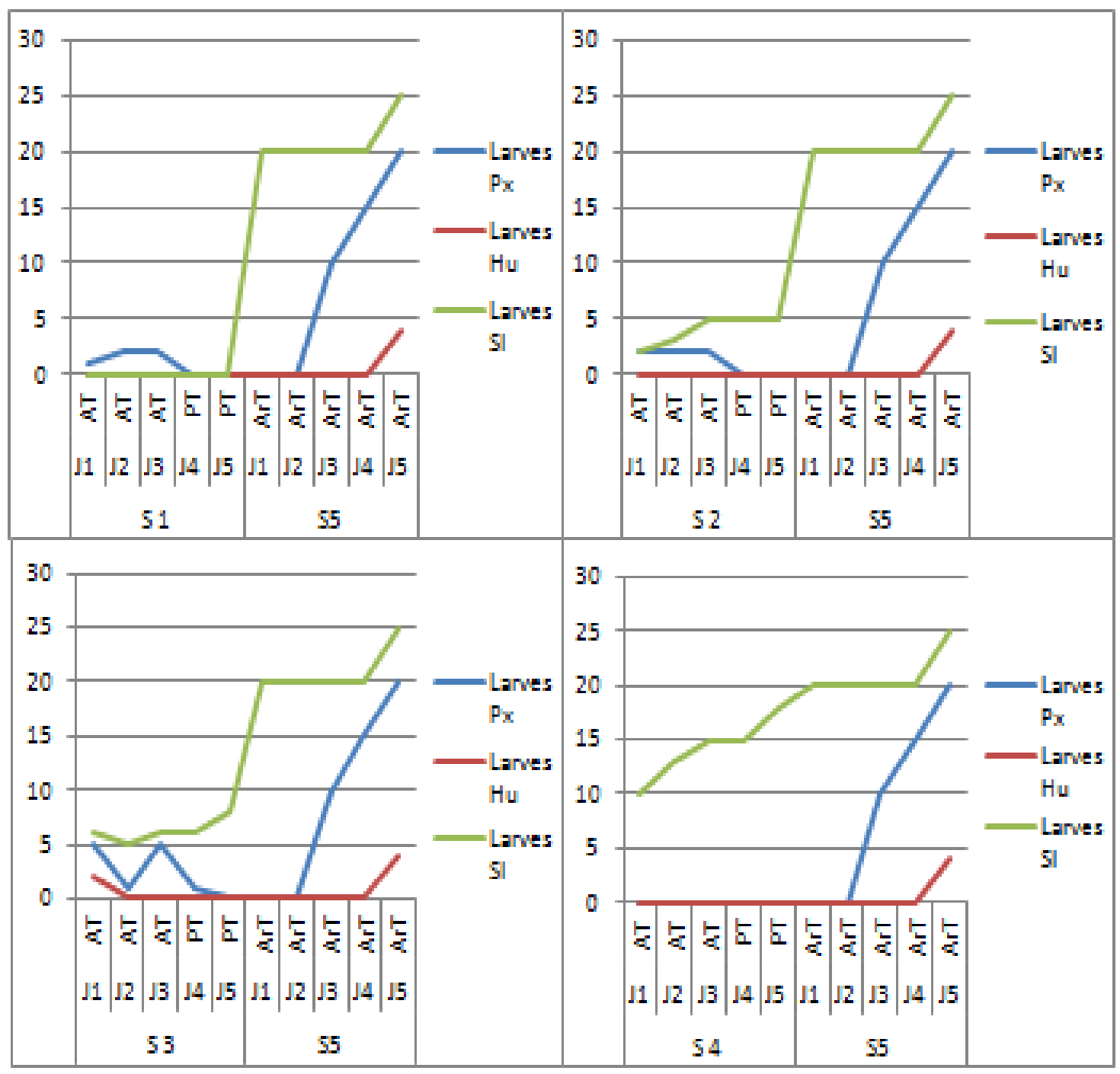

Figure 2: Evolution du nombre de larve en fonction du traitement.

\section{DISCUSSION}

Le but de notre étude était de tester l'effet biocide des extraits aqueux de $C$. procera sur les principaux ravageurs du chou dans la zone des Niayes. Les résultats ont montré que plusieurs insectes dont la plupart nuisibles, sont inventoriés. Les principaux ravageurs rencontrés au cours de l'étude sont : $S$. littoralis, $P$. xylostella, $C$. chalcites, $H$. armigera, $H$. undalis. Il y a aussi la présence de criquets et de pucerons. L'espèce la plus abondante est $S$. littoralis avec une incidence plus élevée que les autres. Nos résultats corroborent ceux de Douan et al. (2013) qui considèrent l'espèce comme ravageur majeur du chou. Une étude sur la distribution spatiotemporelle des principaux lépidoptères de la Menthe verte dans la région de Chaouia a montré une prédominance de l'espèce $C$. chalcites suivie de $S$. littoralis (El fakhouri et al., 2016). Ceci montre que ces espèces sont polyphages et auraient une forte capacité d'adaptation. Toutefois, $P$. xylostella garde son statut de ravageur principal vu l'augmentation significative de sa population pendant la dernière semaine de suivi. Selon Silva-Torres et al. (2010), il est le parasite le plus destructif des crucifères dans le monde entier. Avant 
l'application du traitement, au moment où les plants de chou étaient pratiquement très jeunes, $P$. xylostella était l'espèce la plus abondante dans la parcelle. D'après Sow et al. (2013), les femelles de $P$. xylostella préfèrent les jeunes plantes lors de la ponte. Ceci pourrait s'expliquer par le fait que la teneur en glucosinolate dans la plante avec l'évolution de la pomme constituerait un facteur limitant la ponte des femelles (Sow et al., 2013). Ces observations montrent que l'abondance des larves de $P$. xylostella dépend non seulement $\mathrm{du}$ traitement des parcelles mais également du stade de développement des plants. Les larves de $H$. armigera n'apparaissent qu'à la $3^{\text {ième }}$ semaine de suivi. Ses dégâts sont très remarquables car presqu'une pomme entière est détruite à la $5^{\text {ième }}$ semaine à l'arrêt du traitement(El fakhouri et al., 2016), ont montré que la période d'activité de $H$. armigera est plus courte. Sa présence dans des parcelles peut être due à la proximité des champs test à ceux des tomates compte tenu de la polyphagie de ce ravageur (Vaissayre et al., 1999). Les chenilles de $H$. undalis quant à elles sont moins nombreuses dans les parcelles par rapports aux autres mais leurs dégâts au niveau des pommes étaient non négligeables. Leur présence à la $3^{\text {ième }}$ semaine de suivi a laissé des traces avec la formation de pommes supplémentaires. Nos résultats corroborent ceux de Mewis et al., (2002), qui démontrent que les dommages d'alimentation par une larve simple peuvent avoir comme conséquence l'arrêt du développement et de la mort ou la formation de pommes multiples. Elles causent aussi des dommages majeurs particulièrement dans les jeunes plantes (Mewis et al., 2002).

\section{Effet du traitement sur les principaux ravageurs}

De nos jours, les choix de société se posent à l'ensemble de notre planète, par exemple le développement durable, rendent à nouveau d'actualité l'usage d'extraits de plantes ayant des propriétés insecticides (Mondedji et al., 2014). L'efficacité des extraits de plantes est généralement mesurée à travers l'abondance des populations des ravageurs ou la sévérité des dégâts (Yarou et al., 2017). Les résultats de notre étude ont montré que les extraits aqueux de $C$. procera ont eu un effet sur $P$. xylostella, $H$. undalis, $H$. armigera, $C$. chalcites ainsi que les pucerons rencontrés dans la parcelle en réduisant leurs populations. Avant le début de l'application du traitement 3 jours après le repiquage, quelques larves de $P$. xylostella et une vingtaine de pucerons étaient présents dans les jeunes plantes de chou ayant 24 jours depuis le semis. Ce même phénomène est observé deux semaines plus tard avec les larves de $H$. undalis. Nous avions noté qu'après chaque traitement, le nombre de larves était pratiquement nulle et une réduction significative de la population de pucerons. A la $4^{\text {ième }}$ semaine de traitement, les larves de ces deux ravageurs étaient absentes dans toute la parcelle. Cela peut être dû à l'effet du traitement qui serait néfaste aux ravageurs. Cette efficacité des différents extraits de $C$. procera a été démontrée sur les œufs de $C$. serratus par (Thiaw, 2008). Les extraits de cette plante seraient efficaces sur plusieurs insectes ravageurs. Par contre, pour $S$. littoralis son nombre ne faisait qu'augmenter durant toute la période du test. Ce phénomène nous pousse à penser qu'il résisterait au traitement qui n'a pas un effet significatif (Pvalue $=0,672$ ).

\section{Effet du solvant et de la période de traitement sur les larves}

Les formulations EPM-ERM ne présentent pas une différence significative pour $P$. xylostella, $H$. undalis et $S$. littoralis. En plus les tests deux à deux ne montraient aucune significativité pour les traitements: EPMERM, EPM-EPS et ERM-ERS. La nature des solvants (eau de robinet et eau de puits) n'aurait pas un effet significatif sur l'efficacité des extraits.

\section{Effet du traitement sur la qualité et la maturité des pommes}

Les parcelles non traitées ont eu un niveau d'infestation plus élevé et un rendement moins important. Nos résultats sont similaires à ceux de Sow et al. (2013) qui montrent que les paramètres agronomiques ont été fortement affectés par le niveau d'infestation. Les pommes des parcelles traitées avaient un poids 
et un volume très important qui permet d'être concurrent dans le marché. Selon Mondedji et al. (2014), Les parcelles de chou traitées avec les extraits de plantes donnent les meilleurs rendements par rapport à celui des pesticides chimiques. Un effet accélérateur de la maturation des pommes est noté dans les parcelles traitées par rapport aux témoins. Nous pouvons alors dire que le traitement à base de $C$. procera semble avoir un effet positif sur le rendement et la croissance de la plante.

\section{Conclusion}

La recherche d'alternatives aux insecticides chimiques nous a conduits à tester l'efficacité des extraits aqueux des feuilles de C. procera sur les principaux insectes ravageurs de chou dans la zone des Niayes à Malika. Pour la première fois dans la lutte intégrée, l'utilisation d'un traitement phytosanitaire à base de $C$. procera contre les principaux ravageurs du chou a fait l'objet d'une étude scientifique au Sénégal. Au champ, l'application de l'extrait aqueux des feuilles de $C$. procera a montré sa plus grande efficacité sur les larves de $P$. xylostella, $H$. undalis, $H$. armigera et $C$. chalcites. Il contrôle nettement aussi les pucerons et permettait aux prédateurs d'agir sur la régulation de ces ravageurs en ayant peu d'effet sur eux. Cependant, le traitement n'avait pas un effet remarquable sur les larves de S. littoralis considéré comme une espèce résistante aux extraits de feuilles de $C$. procera. D'après cette étude, nous pouvons dire que les extraits aqueux des feuilles de $C$. procera sont une alternative dans la gestion intégrée des principaux ravageurs du chou. Mais cette pratique doit être approfondie avant d'être considérée dans les programmes de lutte intégrée.

\section{CONFLIT D'INTERETS}

Les auteurs déclarent qu'il n'y a aucun conflit d'intérêt et que l'ordre a été établi à l'unanimité.

\section{CONTRIBUTIONS DES AUTEURS}

SN a été l'expérimentateur principal. TD et MS ont co-encadré le travail sur le terrain et ont activement participé à la rédaction de l'article. BD a participé aussi à la rédaction de l'article.

\section{REFERENCES}

Arvanitakis L. 2013. Interaction entre la teigne du chou Plutella xylostella (L.) et ses principaux parasitoïdes en conditions tropicales: approche éthologique, écologique et évolutive. Thèse de doctorat, Université Paul-Valéry de Montpellier 3, Montpellier, p. 198.

Boisclair J, Estevez B. 2006. Lutter contre les insectes nuisibles en agriculture biologique : intervenir en harmonie face à la complexité. Phytoprotection, 87 (2) : 83-90. DOI: https://doi.org/10.7202/013977ar

Daly P, Desvals L, De-Maleprade M, Deschamps M. 2000. Ravageurs des choux en Nouvelle calédonie: Stratégie de maîtrise des chenilles de quelques lépidoptères. Phytoma-La Défense Des Végétaux, (532): 64-67.

Djomaha ES, Ghogomu TR. 2016. Effet des insecticides, des variétés de chou et des dates de semis sur Plutella xylostella (L.) (Lepidoptera : Plutellidae) dans les hautes terres de l'Ouest Cameroun. International Journal of Biological and Chemical Sciences, 10(3): 1059-1068. DOI: http://dx.doi.org /10.4314/ijbcs.v10i3.13

Douan BG, Doumbia M, Kra KD, Kwadjo E, Martel V, Dagnogo M. 2013. Comparaison de la dynamique des populations de Spodoptera littoralis (Boisduval) (Lepidoptera : Noctuidae) a celles de deux lépidoptères du chou dans le district d'Abidjan en Côte d'Ivoire. $J$. Anim. Plant Sci., 17 (1): 2412 - 2424.

EL-Fakhouri K, Lhaloui S, Faouzi B, Rochd M, EL-Bouhssini M. 2016. Distribution spatio-temporelle des principaux Lépidoptères de la Menthe verte dans la région de Chaouia. Revue Marocaine de Protection des Plantes, (9): 1-10.

FAOSTAT 2013. La situation mondiale de l'alimentation et de l'agriculture 1972. Population (French Edition) 28, p.113.

Gnago J, Danho M, Agneroh T, Fofana I, Kohou A. 2011. Efficacité des extraits de neem (Azadirachta indica) et de papayer 
(Carica papaya) dans la lutte contre les insectes ravageurs du gombo (Abelmoschus esculentus) et du chou (Brassica oleracea) en Côte d'Ivoire. Int. J. Biol. Chem. Sci., 4 (4): 953-966 DOI: http//dx.doi.org/10.4314/ijbcs.v4i4.6303 5

Goalbaye T, Diallo MD, Mahamat-Saleh M, Madjimbe G, Guissé A. 2016. Effet du compost à base de Calotropis procera (Aiton) W.T. Aiton sur la productivité de l'arachide (Arachis hypogaea L.) en zone marginale du Tchad. J. Appl. Biosci., 104: $10034-10041 . \quad$ DOI: http://dx.doi.org/10.4314/jab.v104i1.15

Guèye MT, Seck D, Wathelet JP, Lognay G. 2011. Lutte contre les ravageurs des stocks de céréales et de légumineuses au Sénégal et en Afrique occidentale: synthèse bibliographique. Biotechnol. Agron. Soc. Environ., 15 (1): 183-194.

Labou B. 2016. Distribution des populations de la «Teigne» Plutella xylostella (L), du «Borer» Hellula undalis (F) et des auxiliaires dans les cultures de chou des Niayes au Sénégal. Thèse de doctorat Universite Cheikh Anta Diop de Dakar, Dakar, p.184.

Mawussi G, Kolani L, Devault DA, Alaté KA, Sanda K. 2014. Utilisation de pesticides chimiques dans les systèmes de production maraîchers en Afrique de l'Ouest et conséquences sur les sols et la ressource en eau: Le cas du Togo. 44e congrès du Groupe Français des Pesticides., 46-53.

Mewis I, Kleespies RG, Ulrichs C, Schnitzler WH. 2002. First detection of a microsporidium in the crucifer pest Hellula undalis (Lepidoptera: Pyralidae) a possible control agent. Biological Control., 26: 202-208.

Mondedji AD, Nyamador WS, Amevoin K, Ketoh GK, Glitho IA. 2014. Efficacité d'extraits de feuilles de neem Azadirachta indica (Sapindale) sur Plutella xylostella (Lepidoptera: Plutellidae), Hellula undalis (Lepidoptera: Pyralidae) et Lipaphis erysimi (Hemiptera: Aphididae) du chou Brassica oleracea (Brassicaceae) dans une approche « Champ Ecole
Paysan» au sud du Togo. Int. J. Biol. Chem. Sci., 8 (5): 2286-2295. DOI: http://dx.doi.org/10.4314/ijbcs.v8i5.30

Ngom S, Traore S, Thiam MB, Manga A. 2012. Contamination des produits agricoles et de la nappe phréatique par les pesticides dans la zone des Niayes au Sénégal. Rev. Sci. Technol., 25: 119-130.

Silva-Torres CSA, Pontes IVAF, Torres JB, Barros R. 2010. New Records of Natural Enemies of Plutella xylostella (L.) (Lepidoptera: Plutellidae) in Pernambuco, Brazil. Neotropical Entomology., 39 (5): 835-838.

Son D, Somda I, Legreve A, Schiffers B. 2017. Pratiques phytosanitaires des producteurs de tomates du Burkina Faso et risques pour la santé et l'environnement. Cah. Agric., 26(2): $\quad 1-6 . \quad$ DOI: $10.1051 /$ cagri/2017010

Sow G, Arvanitakis L, Niassy S, Diarra K, Bordat D. 2013. Performance of the parasitoid Oomyzus sokolowskii (Hymenoptera: Eulophidae) on its host Plutella xylostella (Lepidoptera: Plutellidae) under laboratory conditions. International Journal of Tropical Insect Science, 33 (1): 38-45.

Thiaw C. 2008. Bioactivite des extraits de calotropis procera ait. et de senna occidentalis 1. sur Caryedon serratus (ol.), ravageur des stocks et semences d'arachide au Sénégal. Thèse de doctorat, Université cheikh Anta Diop de Dakar, Dakar. p.196.

Touré O, Seck SM. 2005. Exploitations familiales et entreprises agricoles dans la zone des Niayes au Sénégal. Dossier $\mathrm{n}^{\circ}$ 133. Dakar.

Vaissayre M, Martin T, Prudent P, Vassal M. 1999. Prévenir et gérer la résistance des insectes aux pesticides: le cas de la noctuelle Helicoverpa armigera. Agri. Dev., (22): 82-88.

Yarou BB, Silvie P, Komlan FA, Mensah A, Alabi T, Verheggen F, Francis F. 2017. Plantes pesticides et protection des cultures maraîchères en Afrique de l'Ouest (synthèse bibliographique). Biotechnologie, Agronomie, Société et Environnement, 21 (4) : 288-304. 